\title{
Medidas fonológicas em crianças com transtorno fonológico
}

\section{Phonological measures in children with phonological disorders}

\author{
Haydée Fiszbein Wertzner ${ }^{1}$, Gabriele Lopes Claudino², Daniela Evaristo dos Santos Galea ${ }^{3}$, \\ Luciane Kalil Patah ${ }^{4}$, Márcia Mathias de Castro
}

\begin{abstract}
RESUMO
Objetivo: Comparar o desempenho de crianças com e sem transtorno fonológico (TF) quanto às habilidades de consciência fonológica (CF), índice de Porcentagem de Consoantes Corretas - Revisada (PCC-R) e Índice de Inconsistência de Fala (IIF), além de correlacionar estes resultados entre si. Métodos: Participaram 36 sujeitos, entre 5 anos e 7 anos de idade, divididos em: Grupo Pesquisa (GP): 18 crianças com TF; e Grupo Controle (GC): 18 crianças em desenvolvimento típico de linguagem. Foi calculado o PCC-R, aplicado o IIF e o Teste de Sensibilidade Fonológica-Visual (TSF-V): aliteração igual (AI), diferente (AD) e total (AT), rima igual (RI), diferente (RD) e total (RT). Os resultados foram analisados estatisticamente. Resultados: Foram encontradas diferenças na comparação dos grupos em todos os índices, com melhores desempenhos no GC. Neste, houve correlação negativa do IIF com todas as habilidades de CF e com o PCC-R, exceto com RI. Em todos os subtestes do TSF-V houve correlações positivas entre si. No GP, foram encontradas correlações positivas entre o PCC-R e as provas de aliteração; não foram encontradas correlações entre IFF e PCC-R, nem com as provas de CF. Houve correlações no TSF-V: AI com AT; AD com AT; AD com RD; RI com RT e RD com RT. Conclusão: Crianças com TF apresentam pior desempenho; as do GC, na medida em que estabilizam a produção de fala, desenvolvem as habilidades de rima e aliteração. As crianças do GP são mais inconsistentes e parecem desenvolver as habilidades de CF de forma desorganizada.
\end{abstract}

Descritores: Transtornos do desenvolvimento da linguagem; Estudos de linguagem; Índice de gravidade de doença; Testes de linguagem, Criança; Linguagem infantil

\section{INTRODUÇÃO_}

O transtorno fonológico (TF) é uma alteração de fala e linguagem de alta ocorrência na população infantil e sua principal característica é a manifestação inadequada dos sons durante a fala. O TF é heterogêneo e os estudos comprovam a influência de fatores ambientais e genéticos ${ }^{(1-3)}$.

Trabalho realizado no Departamento de Fisioterapia, Fonoaudiologia e Terapia Ocupacional, Faculdade de Medicina, Universidade de São Paulo - USP São Paulo (SP), Brasil.

Conflito de interesses: Não

(1) Departamento de Fisioterapia, Fonoaudiologia e Terapia Ocupacional, Faculdade de Medicina, Universidade de São Paulo - USP - São Paulo (SP), Brasil.

(2) Curso de Fonoaudiologia, Faculdade de Medicina, Universidade de São Paulo - USP - São Paulo (SP), Brasil.

(3) Laboratório de Investigação Fonoaudiológica em Fonologia, Departamento de Fisioterapia, Fonoaudiologia e Terapia Ocupacional, Faculdade de Medicina, Universidade de São Paulo - USP - São Paulo (SP), Brasil.

(4) Programa de Pós-graduação (Mestrado) em Semiótica e Linguística Geral, Departamento de Linguística, Faculdade de Filosofia, Letras e Ciências Humanas, Universidade de São Paulo - USP - São Paulo (SP), Brasil.

(5) Curso de Fonoaudiologia, Universidade Guarulhos - UnG - Guarulhos (SP), Brasil.

Endereço para correspondência: Haydée Fiszbein Wertzner. R. Cipotânea, 51, Butantã, São Paulo (SP), Brasil, CEP: 05360-160. E-mail: hfwertzn@usp.br

Recebido em: 8/8/2011; Aceito em: 19/10/2011
As alterações de fala presentes no TF são verificadas durante o diagnóstico, em que são aplicados testes de linguagem e fala. Após o estabelecimento do diagnóstico, em função da heterogeneidade das alterações há a necessidade de serem realizados testes complementares que auxiliem na verificação das principais dificuldades envolvidas. Assim, medidas fonológicas complementares fornecem informações que auxiliam em tal identificação.

\section{Medidas fonológicas}

Um fator determinante no diagnóstico e planejamento terapêutico da criança com TF é a classificação da gravidade, que pode manifestar-se em diferentes níveis e que indica variações da inteligibilidade de fala no transtorno ${ }^{(1,4)}$.

Há algumas classificações propostas para estabelecer a gravidade do TF, sendo bastante aplicado o índice quantitativo Percentage of Consonants Correct - Revised (PCC-R) ${ }^{(5)}$. Tal índice computa a porcentagem de consoantes corretas, considerando como erros as omissões e substituições.

Outro aspecto muito importante na classificação do TF é a inteligibilidade de fala, que sofre a interferência de vários fatores. Dentre tais fatores, a inteligibilidade é muitas vezes comprometida pela inconsistência de fala (IF), sendo esta definida como produções múltiplas de uma determinada palavra no 
mesmo contexto ${ }^{(6-9)}$. Para constatar a IF é necessário observar tais produções múltiplas, como proposto em estudo em que foram oferecidas às crianças 25 figuras para serem nomeadas três vezes na mesma sessão de avaliação, sendo cada sequência de nomeação separada por uma atividade distratora. A partir das nomeações das figuras, os autores consideraram uma palavra consistente se sua produção ocorreu sempre da mesma forma e inconsistente quando houve múltiplas produções da mesma palavra ${ }^{(6)}$.

Determinar qual o valor de IF que caracteriza o TF ainda é um desafio para os pesquisadores da área, pois durante o desenvolvimento da fala ocorre variabilidade de fala. Alguns estudos indicam que a variabilidade de fala em crianças em desenvolvimento normal da fala é de $13 \%{ }^{(7)}$. Outra pesquisa sugeriu que uma produção com $\mathrm{IF} \geq 40 \%$ é um indicativo de um distúrbio de fala inconsistente, que seria um marcador do $\mathrm{TF}^{(6)}$.

Crianças cuja fala é caracterizada por erros inconsistentes podem ter dificuldade na seleção e sequencialização dos fonemas para a produção da estrutura fonológica, o que indica um déficit no planejamento fonológico com efeitos sobre o planejamento fonético ${ }^{(8)}$.

O Índice de Inconsistência de Fala (IIF) foi desenvolvido para o Português Brasileiro com a finalidade de detectar crianças que apresentam inconsistência de fala ${ }^{(9)}$. Este estudo verificou que crianças do grupo controle $(\mathrm{IFF}=9,8 \%)$ foram mais consistentes do que as crianças com TF (IFF=27,4\%) e detectou efeito de grupo e gênero sobre os resultados. Tais efeitos foram considerados na determinação do valor de corte da IF (curva ROC) e de seus valores de sensibilidade e especificidade. Assim, para as meninas entre 5 anos e 7 anos e 6 meses, o valor de corte da IF foi $21,5 \%$ e, para os meninos da mesma idade foi de $31,9 \%$, enquanto para a idade acima de 7 anos e 6 meses, o valor de corte para as meninas foi de $14,5 \%$, e para os meninos foi de 17,6\%. Esse estudo detectou também que, das 101 crianças avaliadas, 38 (38\%) obtiveram IF acima dos valores de corte estabelecidos, sendo quatro do grupo controle (GC) e 34 do grupo pesquisa (GP). Dentre os sujeitos inconsistentes do GC (8\%), dois eram meninos, entre 5 anos e 7 anos e 6 meses, e duas eram meninas acima de 7 anos e 6 meses. No GP $(67 \%)$ as pesquisadoras encontraram: dez meninos entre 5 anos e 7 anos e 6 meses, e 11 meninos acima de 7 anos e 6 meses; sete meninas entre 5 anos e 7 anos e 6 meses e seis meninas acima de 7 anos e 6 meses.

\section{Consciência fonológica}

A consciência fonológica é a capacidade da criança em identificar e manipular os segmentos sonoros da fala, de forma consciente. É uma das instâncias do processamento fonológico, sendo este definido como o uso da informação fonológica no processamento da linguagem oral e escrita ${ }^{(10,11)}$.

O TF pode afetar tanto a produção como a representação mental dos sons da fala e, dessa forma, prejudicar a articulação e o conhecimento internalizado da língua. Neste último caso, o TF é caracterizado por uma alteração fonêmica, na qual o modo em que a informação sonora é armazenada e representada no léxico mental está afetada. Isso ocorre quando a causa do TF tem uma base linguística ou cognitiva. Assim, o TF pode alterar a consciência sobre os segmentos sonoros da fala e a forma como a informação fonológica é utilizada, trazendo prejuízos para habilidades metafonológicas ${ }^{(12)}$. Consequentemente, crianças com TF apresentam riscos para alterações no desenvolvimento da leitura e da escrita ${ }^{(13,14)}$.

Para avaliar a CF há vários testes desenvolvidos para o Português Brasileiro (PB). Dentre esses, está o Teste de Sensibilidade Fonológica na forma visual (TSF-V) e na forma auditiva $\left(\right.$ TSF-A) ${ }^{(14,15)}$. Tal teste avalia as habilidades de aliteração e rima que correspondem, respectivamente, a segmentos iniciais e finais das palavras. A literatura aponta que, em crianças pré-escolares com desenvolvimento típico de linguagem e fala, as habilidades metafonológicas que envolvem segmentos iniciais são mais fáceis do que aquelas cujos segmentos-alvo estejam no final da palavra ${ }^{(11,15)}$.

O objetivo deste estudo foi comparar o desempenho de crianças com e sem Transtorno Fonológico quanto a habilidades de consciência fonológica, índice de PCC-R na nomeação e Índice de Inconsistência de Fala (IIF). Além disso, busca-se correlacionar os resultados das provas.

\section{MÉTODOS}

A pesquisa foi aprovada pela Comissão de Ética para Análise de Projetos de Pesquisa - CAPPesq, da Diretoria Clínica do Hospital das Clínicas e da Faculdade de Medicina da Universidade de São Paulo (nº 0958/08). Todos os responsáveis assinaram o termo de consentimento livre e esclarecido.

Participaram da pesquisa 36 crianças de ambos os gêneros, cuja faixa etária variou entre 5 anos e 2 meses e 7 anos, sendo 18 sem queixas de alteração de fala e linguagem (Grupo Controle - GC), e 18 com diagnóstico de TF (Grupo Pesquisa-GP).

O GC foi composto por crianças selecionadas de escolas da cidade de São Paulo, que compõe o banco de dados do Laboratório Investigação Fonoaudiológica em Fonologia (LIF-Fonologia), sendo nove sujeitos do gênero feminino e nove do gênero masculino. Os critérios de inclusão destas crianças na pesquisa foram: ausência de queixas de alterações de linguagem e fala, de acordo com os pais e professores, e ter desempenho adequado na prova de fonologia ${ }^{(16)}$ do Teste de Linguagem Infantil ABFW.

O GP foi composto por crianças que procuraram o Laboratório de Investigação Fonoaudiológica em Fonologia, tendo os seguintes critérios de inclusão: idades entre 5 anos e 7 anos e 11 meses; alterações fonológicas na prova de fonologia ABFW; desempenho adequado para a idade nos outros campos da linguagem; avaliação audiológica adequada; não ter sido submetido a tratamento fonoaudiológico anterior e ter o Português como língua materna própria e de seus pais. $\mathrm{O}$ GP foi composto por oito sujeitos do gênero feminino e dez sujeitos do gênero masculino.

Todos os sujeitos foram submetidos às seguintes provas experimentais: prova de nomeação do $\mathrm{ABFW}^{(16)}$, Teste de Inconsistência de Fala ${ }^{(9)}$ e Teste de Sensibilidade Fonológica na forma visual ${ }^{(14,15)}$. A Porcentagem de Consoantes Corretas - Revisada (PCC-R) foi calculada a partir das respostas obtidas na prova de nomeação. As provas foram aplicadas em 
duas sessões, de aproximadamente 45 minutos cada. Para as crianças do GC as provas foram aplicadas em sala silenciosa na escola e para as crianças do GP, foram gravadas em ambiente acusticamente tratado, no LIF-Fonologia.

A análise dos dados respeitou os critérios estabelecidos para cada prova. Para o TSF-V, além dos subtestes Rima e Aliteração igual e diferente, foram computadas também as habilidades totais de aliteração e rima. Assim, Aliteração Total (AT) seria a soma do resultado da Aliteração Igual (AI) com Aliteração Diferente (AD). O mesmo foi realizado para Rima Total (RT), que é o resultado da soma de Rima Igual (RI) e Rima Diferente (RD).

Em relação ao IIF, foram utilizados os valores de corte para o Português Brasileiro( ${ }^{(9)}$, sendo eles: $12,5 \%$ para meninas e $31,9 \%$ para meninos entre 5 anos e 7 anos e 6 meses de idade; e para crianças acima de 7 anos e 6 meses, $14,5 \%$ para meninas e $17,6 \%$ para meninos.

Durante a coleta de dados, um sujeito do GC não compreendeu como deveria ser a execução de nenhuma das tarefas do TSF-V, e um sujeito do GP não compreendeu a execução das tarefas de AD e RD do TSF-V, resultando em perdas amostrais.

Para comparar o desempenho entre os dois grupos nas provas aplicadas, foi utilizado o teste t de Student. A associação entre as provas em cada grupo foi verificada pelo coeficiente de correlação de Spearman, com nível de significância de 5\%.

\section{RESULTADOS}

A análise do IIF indicou haver mais sujeitos inconsistentes no GP do que no GC (Tabela 1). No GC, todas as crianças foram consistentes; já no GP, 56\% foram consistentes e 44\% inconsistentes. Foram encontradas diferenças quando as médias dos grupos em todas as provas foram comparadas, sendo que os melhores desempenhos foram do GC (Tabela 1).

Outro aspecto observado foi que no GC os sujeitos apresentaram maiores valores de PCC-R e menores valores de IIF. Já no GP ocorreu o inverso, os sujeitos apresentaram menores valores de PCC-R e maiores valores de IIF (Figura 1).

Os coeficientes de correlação de Spearman indicaram que no GC houve correlação significativa (negativa) do IIF com todas as provas de consciência fonológica e com o PCC-R, exceto com RI. O PCC-R não apresentou correlação significativa com as demais provas. Neste grupo, em todos os subtestes do TSF-V, houve correlações significativas positivas entre si (Tabela 2).

Para o GP foram encontradas correlações positivas significativas entre o PCC-R e as provas de aliteração, mas não foram encontradas correlações significativas entre o IFF com o PCC-R, nem com as provas de rima e aliteração. Os resultados do TSF-V apresentaram as seguintes correlações significativas: 1) AI com AT; 2) AD com AT; 3) AD com RD; 4) RI com RT e, 5) RD com RT (Tabela 3).

\section{DISCUSSÃO}

$\mathrm{O}$ estudo realizado indicou evidências de diferenças em todos os aspectos analisados (PCC-R calculado para a nomeação do ABFW, IIF e subtestes do TSF-V), sugerindo que as provas aplicadas são eficazes e efetivas na identificação do TF. Quanto à comparação entre os grupos, verificou-se que o GC apresentou melhores desempenhos que o GP, demonstrando

Tabela 1. Comparação entre grupos controle e pesquisa

\begin{tabular}{|c|c|c|c|c|c|c|}
\hline Prova & Grupo & $\mathrm{n}$ & Média & DP & Teste $\mathrm{t}$ & Valor de $p$ \\
\hline \multirow{2}{*}{ PCC-R } & GC & 18 & 0,983 & 0,024 & \multirow{2}{*}{ (34) 6,797 } & \multirow{2}{*}{$\leq 0,001^{*}$} \\
\hline & GP & 18 & 0,742 & 0,148 & & \\
\hline \multirow{2}{*}{ IFF } & GC & 18 & 0,067 & 0,083 & \multirow{2}{*}{ (34) $-3,944$} & \multirow{2}{*}{$0,001^{*}$} \\
\hline & GP & 18 & 0,239 & 0,165 & & \\
\hline TSF-V & $\mathrm{GC}$ & 17 & 9,59 & 1,91 & \multirow{2}{*}{ (33) 3,119} & \multirow{2}{*}{$0,004^{*}$} \\
\hline $\mathrm{Al}$ & GP & 18 & 7,56 & 1,95 & & \\
\hline TSF-V & GC & 17 & 9,35 & 2,26 & \multirow{2}{*}{ (32) 5,338 } & \multirow{2}{*}{$\leq 0,001^{*}$} \\
\hline$A D$ & GP & 17 & 4,76 & 2,73 & & \\
\hline TSF-V & $\mathrm{GC}$ & 17 & 9,471 & 2,027 & \multirow{2}{*}{ (33) 4,759} & \multirow{2}{*}{$\leq 0,001^{*}$} \\
\hline AT & GP & 18 & 6,278 & 1,942 & & \\
\hline TSF-V & $\mathrm{GC}$ & 17 & 8,94 & 2,08 & \multirow{2}{*}{ (33) 6,986 } & \multirow{2}{*}{$\leq 0,001^{*}$} \\
\hline $\mathrm{RI}$ & GP & 18 & 4,28 & 1,87 & & \\
\hline TSF-V & $\mathrm{GC}$ & 17 & 9,47 & 2,03 & \multirow{2}{*}{ (32) 6,635 } & \multirow{2}{*}{$\leq 0,001^{*}$} \\
\hline $\mathrm{RD}$ & GP & 17 & 4,47 & 2,35 & & \\
\hline TSF-V & $\mathrm{GC}$ & 17 & 9,206 & 1,953 & \multirow{2}{*}{ (33) 7,761} & \multirow{2}{*}{$\leq 0,001^{*}$} \\
\hline RT & GP & 18 & 4,361 & 1,739 & & \\
\hline
\end{tabular}

Análise qualitativa e teste $t$ de Student

* Valores significativos $(p \leq 0,05)$

Legenda: $\mathrm{DP}$ = desvio-padrão; $\mathrm{Al}=$ aliteração igual; $\mathrm{AD}=$ aliteração diferente; $\mathrm{AT}=$ aliteração total; $\mathrm{RI}=$ rima igual; $\mathrm{RD}=$ rima diferente; $\mathrm{RT}=$ rima total; IIF = índice de inconsistência de fala; PCC-R = porcentagem de consoantes corretas - revisada; TSF-V = teste de sensibilidade fonológica - visual; GP = grupo pesquisa; GC = grupo controle 
Tabela 2. Coeficientes de correlação entre as variáveis do grupo controle (GC)

\begin{tabular}{|c|c|c|c|c|c|c|c|c|c|}
\hline & & PCC-R & IIF & $\begin{array}{c}\text { TSF-V } \\
\text { Al }\end{array}$ & $\begin{array}{c}\text { TSF-V } \\
\text { AD }\end{array}$ & $\begin{array}{c}\text { TSF-V } \\
\text { RI }\end{array}$ & $\begin{array}{c}\text { TSF-V } \\
\text { RD }\end{array}$ & $\begin{array}{c}\text { TSF-V } \\
\text { AT }\end{array}$ & $\begin{array}{c}\text { TSF-V } \\
\text { RT }\end{array}$ \\
\hline \multirow[t]{3}{*}{ PCC-R } & Coef. corr. & 1,000 & $-0,599$ & 0,292 & 0,164 & 0,051 & 0,222 & 0,228 & 0,175 \\
\hline & Valor de $p$ & & $0,009^{*}$ & 0,256 & 0,529 & 0,845 & 0,391 & 0,378 & 0,501 \\
\hline & $\mathrm{n}$ & & 18 & 17 & 17 & 17 & 17 & 17 & 17 \\
\hline \multirow[t]{3}{*}{ IIF } & Coef. corr. & & 1,000 & $-0,704$ & $-0,540$ & $-0,468$ & $-0,592$ & $-0,630$ & $-0,564$ \\
\hline & Valor de $p$ & & & $0,002^{*}$ & $0,025^{*}$ & 0,058 & $0,012^{*}$ & $0,007^{*}$ & $0,018^{*}$ \\
\hline & $\mathrm{n}$ & & & 17 & 17 & 17 & 17 & 17 & 17 \\
\hline TSF-V & Coef. corr. & & & 1,000 & 0,888 & 0,702 & 0,759 & 0,958 & 0,772 \\
\hline \multirow[t]{2}{*}{ Al } & Valor de $p$ & & & & $\leq 0,001^{*}$ & $0,002^{*}$ & $0,000^{\star}$ & $0,000^{*}$ & $0,000^{*}$ \\
\hline & $\mathrm{n}$ & & & & 17 & 17 & 17 & 17 & 17 \\
\hline TSF-V & Coef. corr. & & & & 1,000 & 0,644 & 0,644 & 0,977 & 0,674 \\
\hline \multirow[t]{2}{*}{$A D$} & Valor de $p$ & & & & & $0,005^{\star}$ & $0,005^{*}$ & $0,000^{*}$ & $0,003^{*}$ \\
\hline & $\mathrm{n}$ & & & & & 17 & 17 & 17 & 17 \\
\hline TSF-V & Coef. corr. & & & & & 1,000 & 0,842 & 0,688 & 0,961 \\
\hline \multirow[t]{2}{*}{$\mathrm{RI}$} & Valor de $p$ & & & & & & $\leq 0,001^{*}$ & $0,002^{*}$ & $0,000^{*}$ \\
\hline & $\mathrm{n}$ & & & & & & 17 & 17 & 17 \\
\hline TSF-V & Coef. corr. & & & & & & 1,000 & 0,711 & 0,951 \\
\hline \multirow[t]{2}{*}{ RD } & Valor de $p$ & & & & & & & $0,001^{*}$ & $0,000^{*}$ \\
\hline & $n$ & & & & & & & 17 & 17 \\
\hline TSF-V & Coef. corr. & & & & & & & 1,000 & 0,734 \\
\hline \multirow[t]{2}{*}{ AT } & Valor de $p$ & & & & & & & & $0,001^{*}$ \\
\hline & $\mathrm{n}$ & & & & & & & & 17 \\
\hline TSF-V & Coef. corr. & & & & & & & & 1,000 \\
\hline
\end{tabular}

* Valores significativos $(p \leq 0,05)$ - Correlação de Spearman

Legenda: $\mathrm{DP}$ = desvio-padrão; $\mathrm{Al}$ = aliteração igual; $\mathrm{AD}$ = aliteração diferente; $\mathrm{AT}$ = aliteração total; $\mathrm{RI}$ = rima igual; $\mathrm{RD}=$ rima diferente; $\mathrm{RT}$ = rima total; IIF = índice de inconsistência de fala; PCC-R = porcentagem de consoantes corretas - revisada; TSF-V = teste de sensibilidade fonológica - visual; Coef. corr. = coeficiente de correlação

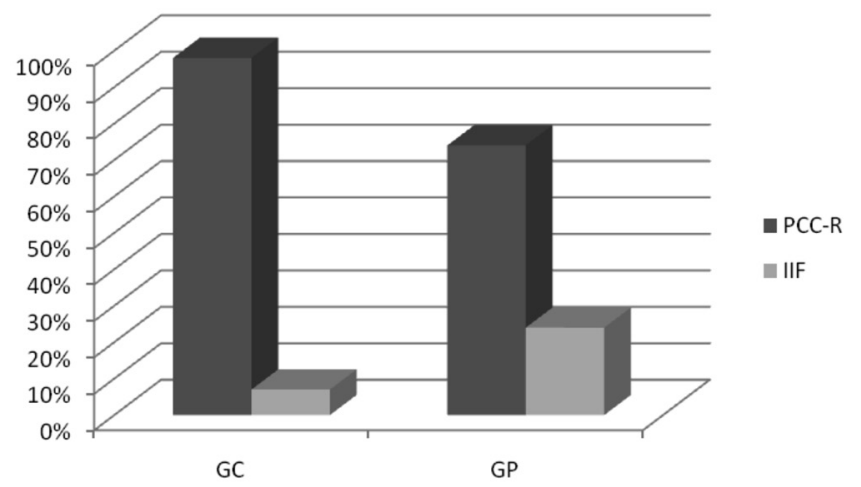

Legenda: GC = grupo controle; GP = grupo pesquisa; IIF = índice de inconsistência de fala; $P C C-R=$ porcentagem de consoantes corretas - revisada

Figura 1. Relação entre PCC-R e IIF nos grupos controle e pesquisa

que as crianças com TF têm alterações fonológicas e inconsistência de fala de graus variados, bem como alterações nas habilidades de rima e aliteração.

Em relação ao índice PCC-R, observou-se que o GP apresentou menores valores que o GC, assim como maior desvio-padrão, confirmando o desempenho fonológico prejudicado e variado das crianças com TF. O PCC-R é uma medida usada para classificar o TF quantitativamente, que tem indicado resultados consistentes em várias línguas ${ }^{(2,5,17-19)}$.

$\mathrm{O}$ fato de todas as crianças do GC terem demonstrado fala consistente, considerando o valor estabelecido para o Português Brasileiro, reforça o fato de que crianças até os 5 anos de idade adquirem a estabilidade do sistema fonológico, ao contrário das crianças com TF, entre as quais $44 \%$ apresentaram inconsistência de fala. A observação da fala mais consistente em crianças com desenvolvimento típico para a idade, e mais inconsistente em crianças da mesma faixa etária com diagnóstico de TF, sugere que as crianças com TF apresentam maior instabilidade na programação fonológica ${ }^{(9)}$.

Durante o desenvolvimento típico, as diferentes produções de uma mesma palavra ocorrem em baixa frequência. Essas inconsistências já foram citadas para o inglês, com índice em torno de $13 \%{ }^{(7)}$, e de $9,8 \%$ para o Português ${ }^{(9)}$. Já nas crianças com diagnóstico de TF a inconsistência de fala ocorre com frequência, e demonstram dificuldade em programar a sequência de sons envolvida na estrutura fonológica em questão, ou seja, os indivíduos selecionam tal estrutura por diferentes caminhos. Esse quadro indica um déficit na programação fonológica com efeitos na programação fonética $^{(8)}$. 
Tabela 3. Coeficiente de correlação entre as variáveis do grupo pesquisa (GP)

\begin{tabular}{|c|c|c|c|c|c|c|c|c|c|}
\hline & & PCC-R & IIF & $\begin{array}{c}\text { TSF-V } \\
\mathrm{Al}\end{array}$ & $\begin{array}{c}\text { TSF-V } \\
\text { AD }\end{array}$ & $\begin{array}{c}\text { TSF-V } \\
\text { RI }\end{array}$ & $\begin{array}{c}\text { TSF-V } \\
\text { RD }\end{array}$ & $\begin{array}{c}\text { TSF-V } \\
\text { AT }\end{array}$ & $\begin{array}{c}\text { TSF-V } \\
\text { RT }\end{array}$ \\
\hline \multirow[t]{3}{*}{ PCC-R } & Coef. corr. & 1,000 & $-0,273$ & 0,556 & 0,562 & 0,289 & 0,041 & 0,609 & 0,094 \\
\hline & Valor de $p$ & & 0,274 & $0,017^{*}$ & $0,019^{*}$ & 0,245 & 0,676 & $0,007^{\star}$ & 0,711 \\
\hline & $\mathrm{n}$ & & 18 & 18 & 17 & 18 & 17 & 18 & 18 \\
\hline \multirow[t]{3}{*}{ IIF } & Coef. corr. & & 1,000 & $-0,450$ & 0,078 & $-0,263$ & $-0,046$ & $-0,119$ & $-0,208$ \\
\hline & Valor de $p$ & & & 0,061 & 0,767 & 0,291 & 0,861 & 0,638 & 0,408 \\
\hline & $\mathrm{n}$ & & & 18 & 17 & 18 & 17 & 18 & 18 \\
\hline TSF-V & Coef. corr. & & & 1,000 & 0,218 & 0,250 & 0,050 & 0,650 & 0,149 \\
\hline \multirow[t]{2}{*}{ Al } & Valor de $p$ & & & & 0,400 & 0,317 & 0,850 & $0,004^{\star}$ & 0,556 \\
\hline & $\mathrm{n}$ & & & & 17 & 18 & 17 & 18 & 18 \\
\hline TSF-V & Coef. corr. & & & & 1,000 & 0,186 & 0,530 & 0,862 & 0,285 \\
\hline \multirow[t]{2}{*}{$A D$} & Valor de $p$ & & & & & 0,474 & $0,035^{\star}$ & $0,000^{*}$ & 0,267 \\
\hline & $\mathrm{n}$ & & & & & 17 & 16 & 17 & 17 \\
\hline TSF-V & Coef. corr. & & & & & 1,000 & 0,471 & 0,371 & 0,843 \\
\hline \multirow[t]{2}{*}{ RI } & Valor de $p$ & & & & & & 0,056 & 0,129 & $0,000^{*}$ \\
\hline & $\mathrm{n}$ & & & & & & 17 & 18 & 18 \\
\hline TSF-V & Coef. corr. & & & & & & 1,000 & 0,469 & 0,837 \\
\hline \multirow[t]{2}{*}{ RD } & Valor de $p$ & & & & & & & 0,058 & $0,000^{*}$ \\
\hline & $\mathrm{n}$ & & & & & & & 17 & 17 \\
\hline TSF-V & Coef. corr. & & & & & & & 1,000 & 0,406 \\
\hline \multirow[t]{2}{*}{ AT } & Valor de $p$ & & & & & & & & 0,095 \\
\hline & $\mathrm{n}$ & & & & & & & & 18 \\
\hline TSF-V & Coef. corr. & & & & & & & & 1,000 \\
\hline
\end{tabular}

* Valores significativos $(p \leq 0,05)$ - Correlação de Spearman

Legenda: $\mathrm{DP}=$ desvio-padrão; $\mathrm{Al}=$ aliteração igual; $\mathrm{AD}=$ aliteração diferente; $\mathrm{AT}=$ aliteração total; $\mathrm{RI}=$ rima igual; $\mathrm{RD}=$ rima diferente; $\mathrm{RT}=$ rima total; IIF = índice de inconsistência de fala; PCC-R = porcentagem de consoantes corretas - revisada; $T S F-V=$ teste de sensibilidade fonológica - visual; Coef. corr. = coeficiente de correlação

A inconsistência pode resultar de diferentes estratégias de controle neural, em vários níveis do sistema de produção, que são refletidos em diferentes padrões de ativação, como a preparação conceitual pré-verbal, a codificação gramatical em integração com o sistema fonológico/fonético, a codificação morfo-fonológica, a seleção fonológica, a codificação fonética, a seleção articulatória, a articulação e a manifestação da fala ${ }^{(20)}$. Desse modo, os achados do presente estudo demonstraram o impacto negativo da inconsistência na fala da criança, na medida em que esta dificulta a aquisição de categorias necessárias para o desenvolvimento de novos fonemas ${ }^{(6)}$.

Outro achado deste estudo foi a correlação negativa entre PCC-R e IIF no grupo controle, indicando que, conforme a criança adquire mais sons corretos, a inconsistência de fala diminui ${ }^{(9)}$. Esta correlação, embora negativa, não foi significativa no GP, mostrando que a programação fonológica pode estar alterada em crianças com TF, independentemente da gravidade. Tal resultado pode ter sido influenciado pela idade das crianças, pois em outro estudo com crianças com TF falantes do Português Brasileiro, entre a faixa etária de 5 a 10 anos de idade, houve associação entre os valores do IIF e a gravidade medida pelo PCC- $\mathrm{R}^{(21)}$.
Quanto às habilidades de CF testadas no presente estudo, as crianças do GP apresentaram desempenho inferior quando comparadas com crianças do GC, demonstrando que as crianças com TF têm dificuldades nas habilidades de $\mathrm{CF}^{(12,14,22-24)}$. Tais dificuldades podem ser provenientes de alterações no conhecimento internalizado da língua, que muitas vezes está afetado em crianças com $\mathrm{TF}^{(23,24)}$.

Outro estudo ${ }^{(25)}$ relatou a importância do desenvolvimento da rima infantil para o processo de alfabetização, sugerindo que a reflexão sobre a estrutura da palavra, e não somente o significado como um todo, é fundamental para o sucesso em tarefas de segmentação, que por sua vez, são essenciais para o desenvolvimento das demais habilidades de CF. Tal fator explica o fracasso de crianças com TF durante o processo de alfabetização.

Em função dessas dificuldades, estudos sugerem que o diagnóstico de TF seja realizado o quanto antes e tratado adequadamente, visto que frequentemente crianças com TF têm alterações nas habilidades de CF e quando expostas à alfabetização, apresentam mais dificuldades de leitura e escrita ${ }^{(13,23,24)}$.

As dificuldades de crianças com TF em habilidades de $\mathrm{CF}$ refletem as relações de percepção de fala e os gestos articulatórios. Isso ocorre em função das falhas quanto às representações 
fonológicas, que podem prejudicar o uso da informação fonológica para manipular segmentos das palavras ${ }^{(12,23-26)}$.

As diferenças entre GC e GP ocorreram em todas as provas, embora em pesquisa brasileira com TSF-V ${ }^{(14)}$ tenha-se observado diferença significativa entre crianças com e sem TF somente na prova de aliteração diferente. Entretanto, em todas as provas, as crianças com TF apresentaram menos acertos. É importante ressaltar que nesta pesquisa foram estudadas crianças entre 7 anos e 10 anos de idade, o que pode ter influenciado no resultado encontrado.

Analisando a correlação entre as provas em cada grupo estudado, foi possível observar que no GC houve maior número de correlações entre as provas, do que no GP. Nota-se que no GC, para o PCC-R não houve correlação com as provas do TSF-V, porém, é preciso destacar que os valores de PCC-R nesse grupo foram próximos do valor máximo, com pouca variabilidade. Portanto, embora apresentassem bom desempenho no sistema fonológico, ainda estavam em desenvolvimento das habilidades de CF avaliadas no TSF$-\mathrm{V}^{(14,23,24,27)}$.

Já no GP, foram encontradas correlações significativas do PCC-R com as habilidades de aliteração, mas não com as habilidades de rima. É importante destacar que a heterogeneidade do TF pode explicar parcialmente os achados, pois outros estudos também tiveram resultados semelhantes ${ }^{(23,24,28)}$. Outra questão a ser apontada é que as habilidades de aliteração são consideradas na literatura como as mais fáceis e adquiridas mais cedo pelas crianças ${ }^{(11,15)}$.

Além disso, há que se considerar que a correlação positiva entre PCC-R e aliteração, no GP, pode ser explicada pelo fato que, à medida que o PCC-R aumenta, as crianças têm mais opções de consoantes disponíveis. Desta forma, o aumento de consoantes corretas aprimora as representações fonológicas, que são essenciais para a manipulação dos segmentos das palavras. Porém, há indício de que este aperfeiçoamento não seja suficiente para reparar os danos causados em rima que, conforme mostra a literatura, é mais difícil de ser realizada, se comparada à aliteração $0^{(11,15)}$.

O fato da IIF na criança com TF (GP), com até 7 anos de idade, não manifestar correlação significativa com as habilidades de consciência fonológica, sugere que tanto a representação fonológica, necessária para o bom desempenho nas habilidades de aliteração e rima, como a programação fonológica e fonética, necessárias para produzir uma mesma palavra de forma consistente, estão alteradas, o que impossibilita a criança de realizar as tarefas com organização.

Vários estudos na literatura apontam resultados variados quanto à questão da relação entre gravidade, IIF e habilidades de $\mathrm{CF}^{(23,24)}$. Em estudo realizado com crianças com $\mathrm{TF}^{(21)}$ foi verificado que maiores valores de PCC-R estão associados a menores valores de IIF de fala, ou seja, quanto mais grave a manifestação do TF, parece haver maior dificuldade na programação fonológica.

Estudo recente ${ }^{(8)}$ apontou que crianças com TF e inconsistência de fala com leitura e habilidades de rima e aliteração sem comprometimento demonstraram representação fonológica adequada, bem como bom controle motor-oral. A dificuldade está então centrada na seleção e sequenciação dos fonemas (programação fonológica) de uma palavra ou enunciado.

De forma geral, as crianças com TF apresentam riscos para as alterações de CF e posteriormente para a leitura e a escrita $^{(23,24,29)}$.

Estudos demonstram que os processamentos cognitivo-linguístico, motor da fala e auditivo se interrelacionam em crianças com desenvolvimento de linguagem e fala típicos para a idade. Já nas crianças com TF, que podem apresentar maior dificuldade em algum dos processamentos, a interrelação reflete-se em déficit em várias habilidades, manifestando-se, como no presente estudo, em menos consoantes corretas, mais inconsistência de fala e alterações na consciência fonológica, as quais podem levar a futuras dificuldades escolares ${ }^{(8,20,29)}$.

Os resultados obtidos indicam que as provas de IIF, TSF-V e o índice PCC-R mostraram-se eficazes para identificar crianças com TF. Houve também evidências de que os procedimentos aplicados se complementam, fornecendo importantes informações ao fonoaudiólogo no diagnóstico do TF.

\section{CONCLUSÃO_}

As crianças portadoras de $\mathrm{TF}$ apresentam pior desempenho quanto ao PCC-R, IIF e habilidades de CF, quando comparadas com as crianças sem TF. Estas, na medida em que estabilizam a produção de fala, desenvolvem as habilidades de rima e aliteração. No GP, as crianças mais graves, também são mais inconsistentes e desenvolvem as habilidades de CF de forma desorganizada.

Nas crianças do GC há correlação positiva e significativa entre a maioria das provas, porém nas do GP observam-se poucas correlações, o que sugere desempenho mais heterogêneo nas provas aplicadas. As crianças inconsistentes variam quanto à gravidade e quanto às habilidades de consciência fonológica de rima e aliteração.

Os procedimentos aplicados são efetivos para indicar as dificuldades das crianças com TF, além de evidenciar as diferenças entre elas e crianças sem TF.

\section{AGRADECIMENTOS}

A pesquisa realizada recebeu apoio da Fundação de Amparo à Pesquisa do Estado de São Paulo (FAPESP) n²008/571452 (primeira autora) e bolsa de Iniciação Científica 2010/007754 (segunda autora). 


\begin{abstract}
Purpose: To compare and correlate the performance of children with and without phonological disorders (PD) according to phonological awareness (PA) abilities, Percentage of Consonants Correct - Revised (PCC-R) and Speech Inconsistency Index (SII). Methods: Participants were 36 children with ages between 5 and 7 years divided into: Research Group (RG) - 18 children with PD; and CG - 18 typically developing children. The PCC-R was calculated, and the SII and the Phonological Sensitivity Test - Visual mode (PST-V) were applied. The PST-V consists of six tasks: equal and different alliteration (EA and DA, respectively), total alliteration (TA), equal and different rhyme (ER and DR, respectively), and total rhyme (TR). Results were statistically analyzed. Results: Differences were found between groups in all indexes, with better performances of the CG. In this group there were negative correlations between SII and all PA abilities and between SII and PCC-R, except for the ER. There were positive correlations between all PST-V subtests. On the RG, positive correlations were observed between PCC-R and alliteration abilities; no correlations were found between SII and PCC-R nor between SII and PA subtests. There were correlations between PST-V abilities: EA and TA; DA and TA; DA and DR; ER and TR; DR and TR. Conclusion: Children with PD had worse performances. CG children develop rhyme and alliteration abilities as they stabilize their speech production. RG children are more inconsistent and tend to develop PA abilities in a more disorganized manner.
\end{abstract}

Keywords: Language development disorders; Language arts; Severity of illness index; Language tests, Child; Child language

\section{REFERÊNCIAS}

1. Lewis BA, Freebairn LA, Habseb AJ, Stein CM, Shriberg LD, Iyengar SK, Gerry Taylor H. Dimensions or early speech sound disorders: a factor analytic study. J Commun Disord. 2006;39(2):139-57.

2. Wertzner HF, Ramos AC, Amaro L. Índices fonológicos aplicados ao desenvolvimento fonológico típico e ao transtorno fonológico. Rev Soc Fonoaudiol. 2004;9(4):199-203.

3. Shriberg LD, Lewis BA, Tomblin JB, McSweeny JL, Karlsson HB, Scheer AR. Toward diagnostic and phenotype markers for genetically transmitted speech delay. J Speech Lang Hear Res. 2005;48(4):834-52.

4. Shriberg LD. Four new speech and prosody-voice measures for genetics research and other studies in developmental phonological disorders. J Speech Hear Res. 1993;36(1):105-40.

5. Shriberg LD, Austin D, Lewis BA, McSweeny JL, Wilson DL. The speech disorders classification system (SDCS): extensions and lifespan reference data. J Speech Lang Hear Res. 1997;40(4):723-40.

6. Dodd B, Holm A, Crosbie S, McComarck P. Differential diagnosis and treatment of children with speech disorder. London: Whurr Publishers; 2005.

7. Holm A, Crosbie S, Dodd B. Differentiating normal variability from inconsistency in children's speech: normative data. Int J Lang Commun Disord. 2007;42(4):467-86.

8. McIntosh B, Dodd B. Evaluation of core vocabulary intervention for treatment of inconsistent phonological disorder: three treatment case studies. Child Lang Teach Ther. 2008;25(1):9-29.

9. de Castro MM, Wertzner HF. Speech inconsistency index in Brazilian Portuguese-speaking children. Folia Phoniatr Logop. 2011;63(5):23741.

10. Santamaria VL, Leitão PB, Assencio-Ferreira VJ. A consciência fonológica no processo de alfabetização. Rev CEFAC. 2004;6(3):23741.

11. Puffpaff LA. A developmental continuum of phonological sensitivity skills. Psychol Sch. 2009;46(7):679-91.

12. Marchetti PT, Mezzomo CL, Cielo CA. Desempenho em consciência silábica e fonêmica em crianças com desenvolvimento de fala normal e desviante. Rev CEFAC. 2010;12(1):12-20.

13. Wagner R, Torgesen J, Rashotte C. Comprehensive test of phonological processing (CTOPP). Austin: Proed; 1999.

14. Herrero SF. Desempenho de crianças com transtorno fonológico no teste de sensibilidade fonológica e de leitura e escrita [tese]. São Paulo: Universidade de São Paulo, Faculdade de Filosofia, Letras e Ciências Humanas; 2007.

15. Herrero SF. Perfil das crianças pré-escolares e escolares no teste de sensibilidade fonológica [dissertação] São Paulo: Universidade de São Paulo, Faculdade de Filosofia, Letras e Ciências Humanas; 2001.

16. Wertzner HF. Fonologia. In: Andrade CR, Befi-Lopes DM, Fernandes FD, Wertzner HF. ABFW: teste de linguagem infantil nas áreas de fonologia, vocabulário, fluência e pragmática. Barueri: Pró-Fono; 2004.

17. Wertzner HF, Galea DES. Características fonológicas de crianças com e sem transtorno. Rev Soc Fonoaudiol. 2002;7(1):44-50.

18. Wertzner HF, Amaro L, Galea DE. Phonological performance measured by speech severity indexes related to correlated factors. São Paulo Med J. 2007;125(6):309-14.

19. Campbell TF, Dollaghan C, Janosky JE, Adelson PD. A performance curve for assessing change in Percentage of Consonants Correct Revised (PCC-R). J Speech Lang Hear Res. 2007;50(4):1110-9.

20. Munhall KG. Functional imaging during speech production. Acta Psychol (Amst). 2001;107(1-3):95-117.

21. Castro MM, Wertzner HF. Estimulabilidade e inconsistência de fala como provas complementares ao diagnóstico do transtorno fonológico. In: $18^{\circ}$ Congresso Brasileiro de Fonoaudiologia; 2010 Set 22-25; Curitiba.

22. Gillon GT. Effective practice in phonological awareness intervention for children with speech sound disorder. Perspect Lang Learn Educ. 2007;14:18-23.

23. Rvachew S, Grawburg M. Correlates of phonological awareness in preschoolers with speech sound disorders. J Speech Hear Res. 2006;49(1):74-87.

24. Raitano NA, Pennington BF, Tunick RA, Boada R, Shriberg LD. Preliteracy skills of subgroups of children with speech sound disorders. J Child Psychol Psychiatry. 2004;45(4):821-35.

25. Stackhouse J. Persisting speech difficulties, spelling, and phonological awareness. Perspect Lang Learn Educ. 2007;14:5-10.

26. Morales MV, Mota HB, Keske-Soares M. Consciência fonológica: desempenho de crianças com e sem desvios fonológicos evolutivos. Pró-Fono. 2002;14(2):153-64.

27. Rvachew S, Chiang PY, Evans N. Characteristics of speech errors produced by children with and without delayed phonological awareness skills. Lang Speech Hear Serv Sch. 2007;38(1):60-71.

28. Larrivee LS, Catts HW. Early reading achievement in children with expressive phonological disorders. Am J Speech Lang Pathol. 1999;8:118-28.

29. Holm A, Farrier F, Dodd B. Phonological awareness, reading accuracy and spelling ability of children with inconsistent phonological disorder. Int J Lang Commun Disord. 2008;43(3):300-22. 